\title{
Embarazo ectópico en el abdomen agudo obstétrico
}

\section{Ectopic pregnancy in the obstetric acute abdomen}

\section{Embarazo ectópico en el abdomen agudo obstétrico}

\author{
Carlos A. Vizueta-Chávez ${ }^{\text {I }}$ \\ carlos.vizuetac@ug.edu.ec \\ Byron O. López-Silva II \\ byron.lopezs@ug.edu.ec \\ Jorge E. Balon-Benavides III \\ jorgebalonbe@gmail.com \\ Rosario H. Zambrano-Bonilla IV \\ rosariohz@gmail.com
}

Recibido: 30 de enero de 2017 * Corregido: 20 de febrero de $2017 *$ Aceptado: 20 junio de 2017

\footnotetext{
Médico, Universidad de Guayaquil; Clínica Kennedy, Guayaquil, Ecuador. Médico, Universidad de Guayaquil, Guayaquil, Ecuador. Médico, Universidad de Guayaquil, Guayaquil, Ecuador. Médico, Universidad de Guayaquil, Guayaquil, Ecuador.
} 


\section{Resumen}

El embarazo ectópico como causa determinante en el abdomen agudo obstétrico, que de acuerdo de las estadísticas internacionales va en incremento y en nuestro medio de igual forma. Ya que se tomaron como muestra a 389 casos registrados, que cumplían con los requisitos de inclusión en el Hospital Gineco Obstétrico Enrique Sotomayor de la ciudad de Guayaquil 2014 y 2015. Este estudio retrospectivo se lo realizo en el desarrollo de una tesis de titulación cuyo actor y tutor estamos en este artículo y que por la valiosa investigación detallada que se llevó de acuerdo a una previa revisión bibliográfica Gineco-obstétrica, con protocolos de emergencia obstétrica y la ayuda de medicina con datos estadísticos internacionales (Los Centers for Disease Control and prevention) y nuestras estadísticas nacionales dadas por el INEC no actualizadas. Con esta muestra institucional vemos que al momento estamos en iguales condiciones con los centros obstétricos internacionales que presenta un aumento que es preocupante porque incrementa el problema de salud a mediano y largo plazo, mucho más cuando no se hace diagnostico a tiempo. Si bien es cierto que ha disminuido el índice de morbimortalidad, también es cierto que sea incrementado el número de embarazos ectópicos como se observa en este estudio. Ni aun teniendo en nuestro medio métodos clínicos para tratar el embarazo ectópico como el uso de metrotexato a sus debidos tiempos y no esperar llegar a una emergencia obstétrica, como también se demuestra en el estudio con cuadros clínicos hemorrágicos y hipovolémicos y terminar con una acción quirúrgica (salpingectomia) y otros que en muchas ocasiones tienen diagnostico reservado.

Palabras clave: Ectópico; implantación; emergencia. 


\section{Abstract}

Ectopic pregnancy as a determining cause in the acute obstetric abdomen, which according to international statistics is increasing and in our environment in the same way. As a sample of 389 registered cases, which complied with the requirements of inclusion in the hospital-obstetrical Gineco-obstetrics Enrique Sotomayor

of the city of Guayaquil 2014 and 2015. This retrospective study was carried out in the development of a thesis titled whose actor and guardian we are in this article and that for the valuable detailed investigation that was carried out according to a previous bibliographical revision Gyneco-obstetric, with obstetric emergency protocols and the medical help with international statistical data (The Centers for Disease Control and prevention) and our national statistics given by the INEC not updated. With this institutional sample we see that at the moment we are in the same conditions with the international obstetric centers that presents an increase that is worrisome because it increases the health problem in the medium and long term, much more when it is not done diagnosis in time. Although it is true that the morbidity and mortality rate has decreased, it is also true that the number of ectopic pregnancies is increased as observed in this study. Not even in our midst clinical methods to treat ectopic pregnancy as the use of methotrexate in its due time and not expect to reach an obstetric emergency, as is also demonstrated in the study with hemorrhagic and hypovolemic clinical signs and end with a surgical action (salpingectomy) and others who in many cases have a reserved diagnosis.

Key words: Ectopic; implantation; emergency. 


\section{Introducción.}

Las tasas de embarazo ectópico han aumentado durante los últimos 30 años a nivel mundial, tanto en países del primer mundo como en países subdesarrollados, es más frecuente encontrar en la consulta médica mujeres en edad fértil, activas sexualmente y con factores de riesgo de un embarazo ectópico, que consultan por amenorrea, así como en las emergencias encontrar casos de abdomen agudo de tipo obstétrico en el que la causa más común sea la ruptura de un embarazo ectópico ${ }^{1-3}$.

Los Centers for Disease Control and prevention (CDC) indican que la incidencia del embarazo ectópico en Estados Unidos se incrementó significativamente en los últimos 30 años ${ }^{4}$.

Esta tendencia no es sólo de los países del primer mundo, también encontramos en los otros países de América mayores tasas de embarazo extrauterinos, así como un aumento en nuestro país ${ }^{5-}$ 6.

Se manejan varias teorías que tratan de explicar el aumento en la incidencia de los embarazos ectópicos; aumento en la incidencia de salpingitis y enfermedades de transmisión sexual, mujeres añosas, tratamientos de fertilidad, anticoncepción fallida, mejor diagnóstico precoz, etc. En la actualidad el embarazo extrauterino es la principal causa de muerte en el primer trimestre del embarazo ${ }^{4}$

Sin embargo en nuestro país aun no contamos con un estudio real, sobre esta problemática de salud, en el Hospital Gineco Obstétrico Enrique Sotomayor de la ciudad de Guayaquil se realizó el estudio de la tendencia en los años 2014 y 2015, que nos permitio identificar la morbimortalidad, grupos etarios, sitios mas frecuentes de implantacion y tratamiento utilizado. 
La presente investigacion pretende descrbir el Embarazo Ectópico dentro del abdomen agudo, su etiológia, factores de riesgo, manifestaciones clinicas, métodos diagnóstico y tratamiento del embarazo ectópico en el primer mundo (E.U, Europa) en nuestro país ((INEC), 2014) y en el Hospital Gineco Obstétrico Enrique Sotomayor, de esta manera contribuyendo con datos actualizados de esta patología, sirviendo de base para futuras investigaciones sobre el tema.

Como objetivo tenemos el establecer las manifestaciones clínicas, los principales factores de riesgo y el tratamiento de las pacientes que se atendieron por embarazo ectópico y/o abdomen agudo obstétrico en el Hospital Gineco-Obstétrico Enrique Sotomayor en los años 2014 y 2015.

\section{Materiales y métodos.}

La investigación tiene el carácter explorativo, descriptivo, explicativo, con un enfoque cuantitativo, analítico y comparativo cogiendo como fuente el análisis de las historias clínicas proporcionadas por el Hospital Gineco Obstétrico Enrique Sotomayor, de las pacientes que acudieron a esta casa de salud en el año 2014 y 2015.

Como parte inicial se procedió a la investigación analítica sobre el tema de embarazo ectópico en el abdomen agudo, sus factores de riesgo más importantes, las etnias de mayor impacto, el grupo poblacional más afectado, así como los sitios de implantación y el tratamiento recibido en nuestro país y a nivel mundial, con la finalidad de preservar la salud sexual y reproductiva.

Una estadística real de embarazos extrauterinos se hace difícil, ya que afortunadamente para las gestantes los embarazos ectópicos no complicados pueden resolverse sin dar gran sintomatología (se reabsorben), sin embargo se ve un aumento de casos de embarazos ectópicos. 
El diseño de la investigación es de tipo analítico, estadístico y comparativo con el fin de graficar los valores obtenidos, con base en las variables se realizara el analizis para poder ver los factores que se han visto aumentados con respecto a la causa, la comorbilidad, medidas terapéuticas empleadas y los resultados obtenidos. Con esto se desea tener un mayor cuadro de análisis con respecto a la prevención, seguimiento y tratamiento frente a casos de embarazo ectópico.

La ejecución del estudio se realizó en dos fases:

1. La primera fase tuvo una duración de dos meses, en la que se realizó la recolección de datos que el departamento de estadísticas proporcionó de las historias clínicas.

2. La segunda fase tuvo una duración de dos meses que consistió en la revisión, tabulación y evaluación de la información

La recolección de datos fue realizada con las historias clínicas de las mujeres diagnosticadas de embarazo ectópico y abdomen agudo obstétrico desde enero del año 2014 hasta diciembre del año 2015, donde se utilizó datos estadísticos del INEC, exámenes de laboratorio y reportes de ecografías para la realización de la estadificaión de resultados.

Se estudió 389 pacientes de raza mestiza que acudieron al Hospital Ginecológico Enrique Sotomayor de la ciudad de Guayaquil en los años 2014 - 2015 por presentar un cuadro clínico de abdomen agudo de tipo obstétrico cuyas edades oxcilan entre los 17 y 40 años, en base a las variables se obtuvo los siguientes resultados: 
En el año 2014 encontramos 201 pacientes:

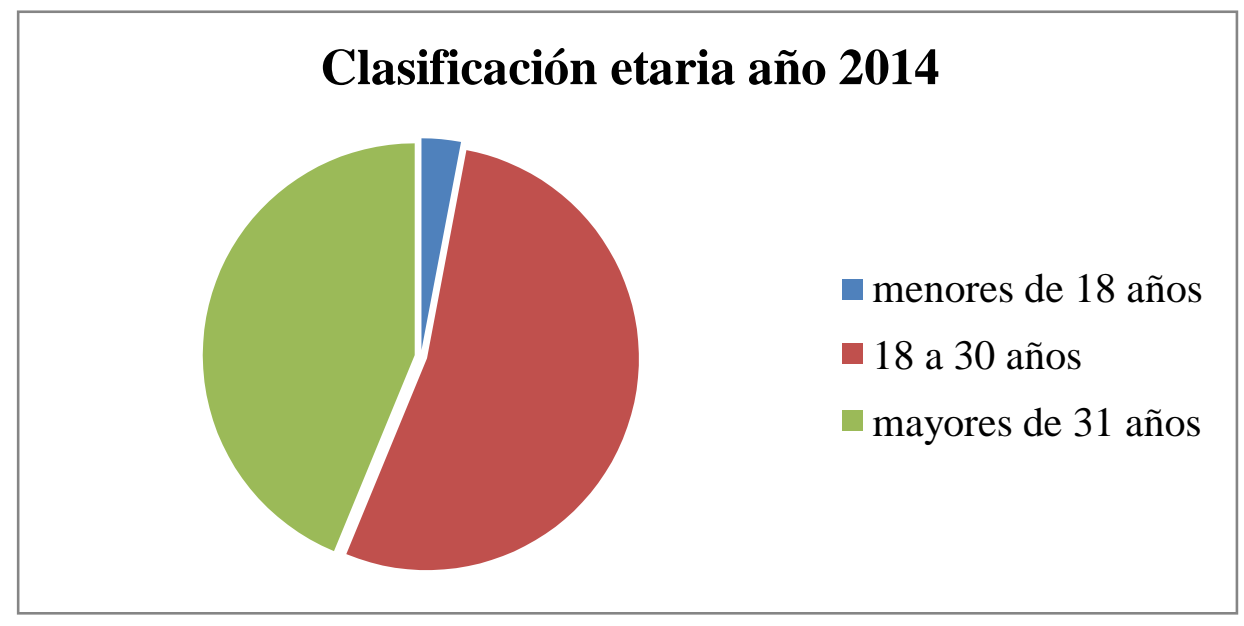

\section{Grafico $N^{\circ}$ 1.- Grupo etario}

- 6 son menores de 18 años corresponde al 3\%.

- 107 están entre los 18 a 30 años de edad corresponde al 53\%.

- 88 son mayores de 31 años, corresponde al $44 \%$.

\begin{tabular}{|l|l|}
\hline OCUPACIÓN AÑO 2014 Cantidad \\
\\
$\square$ Quehaceres domesticos \\
$\square$ Empleada publica \\
$\square$ Empleada privada \\
$\square$ Estudiante \\
$\square$ Trabajo independiente \\
\end{tabular}

Grafico $N^{\circ}$ 2.- Ocupación 
- Quehaceres domésticos:149 (74\%)

- Empleada pública: $5(2 \%)$

- Empleada privada: $15(7 \%)$

- Estudiante: $6(3 \%)$

- Trabajo independiente: $26(26 \%)$

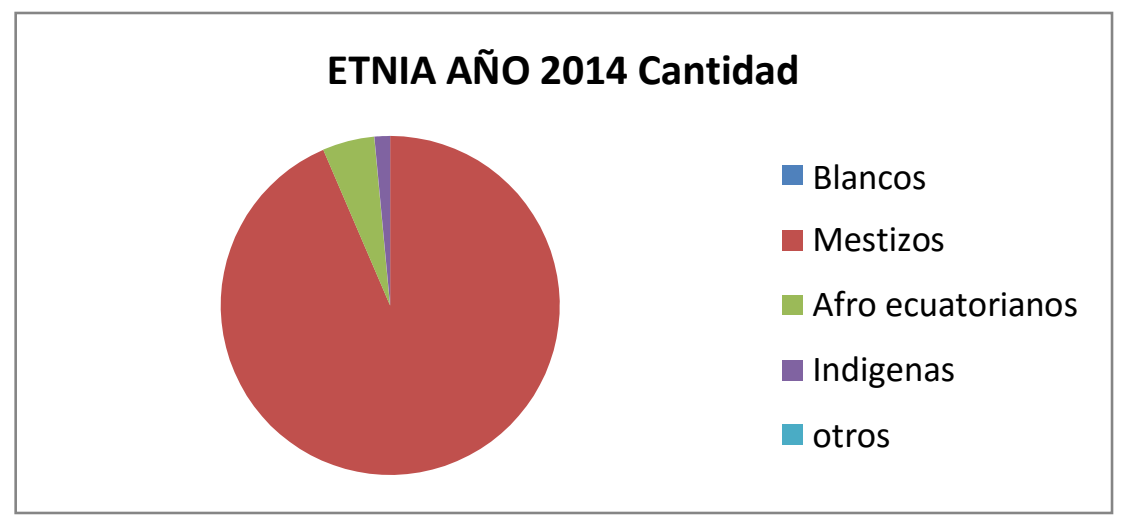

Grafico $N^{\circ}$ 3.- Etnia

- $\quad$ Blancos: $0(0 \%)$

- $\quad$ Mestizos: $188(94 \%)$

- $\quad$ Afro ecuatorianos: $10(5 \%)$

- Indígenas: $3(1 \%)$

- Otros: $0(0 \%)$ 


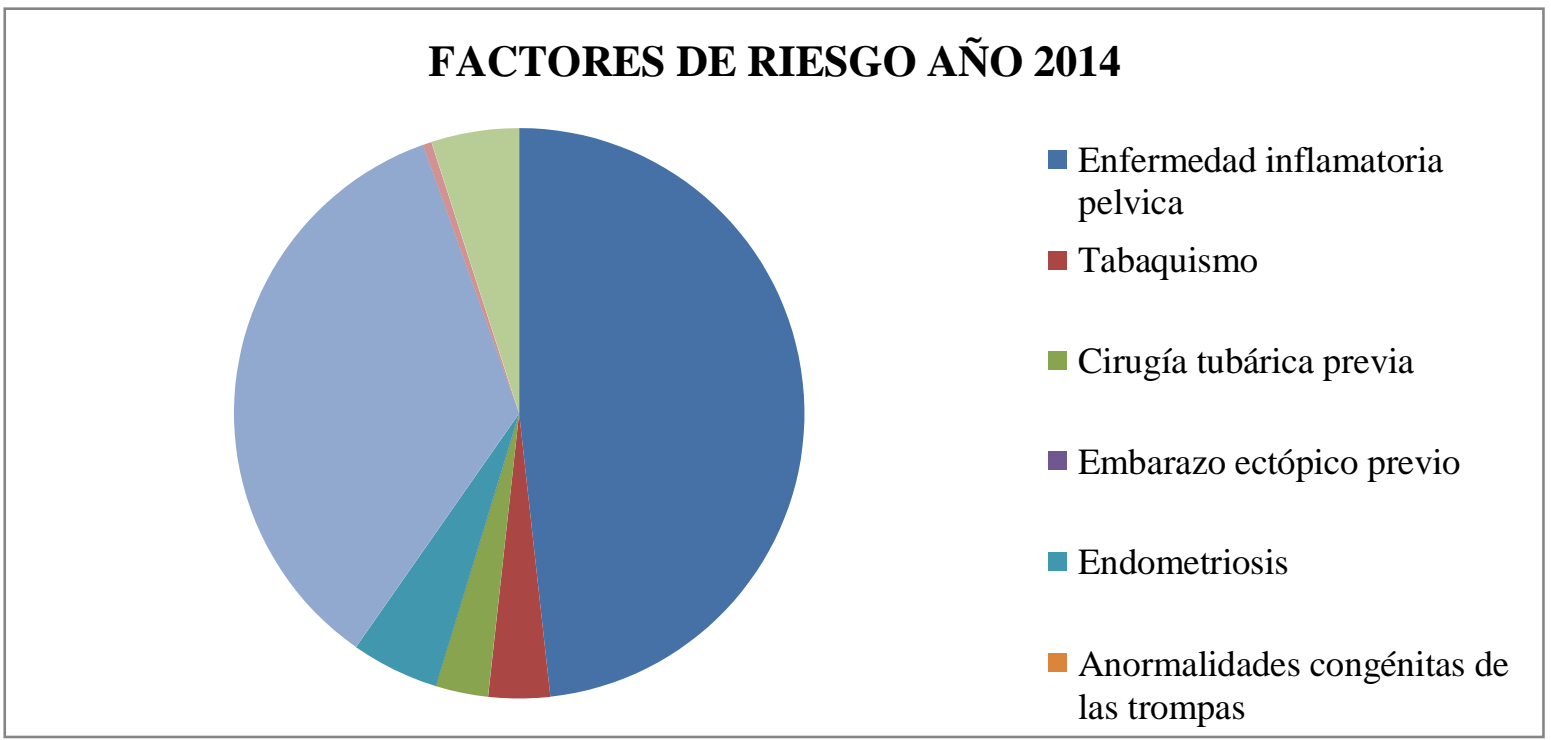

\section{Grafico $N^{\circ}$ 4.- Factores de riesgo}

- Enfermedad inflamatoria pelvica: 97 (49\%)

- Tabaquismo: 7 (3\%)

- Cirugía tubárica previa: 6 (3\%)

- Embarazo ectópico previo: $0(0 \%)$

- Endometriosis: 10 (5\%)

- Anormalidades congénitas de las trompas: $0(0 \%)$

- Dispositivos intrauterinos: 70 (35\%)

- Fertilización in vitro e inducción de la ovulación: 1 (0\%)

- Salpingitis ístmica nodosa: 10 (5\%) 


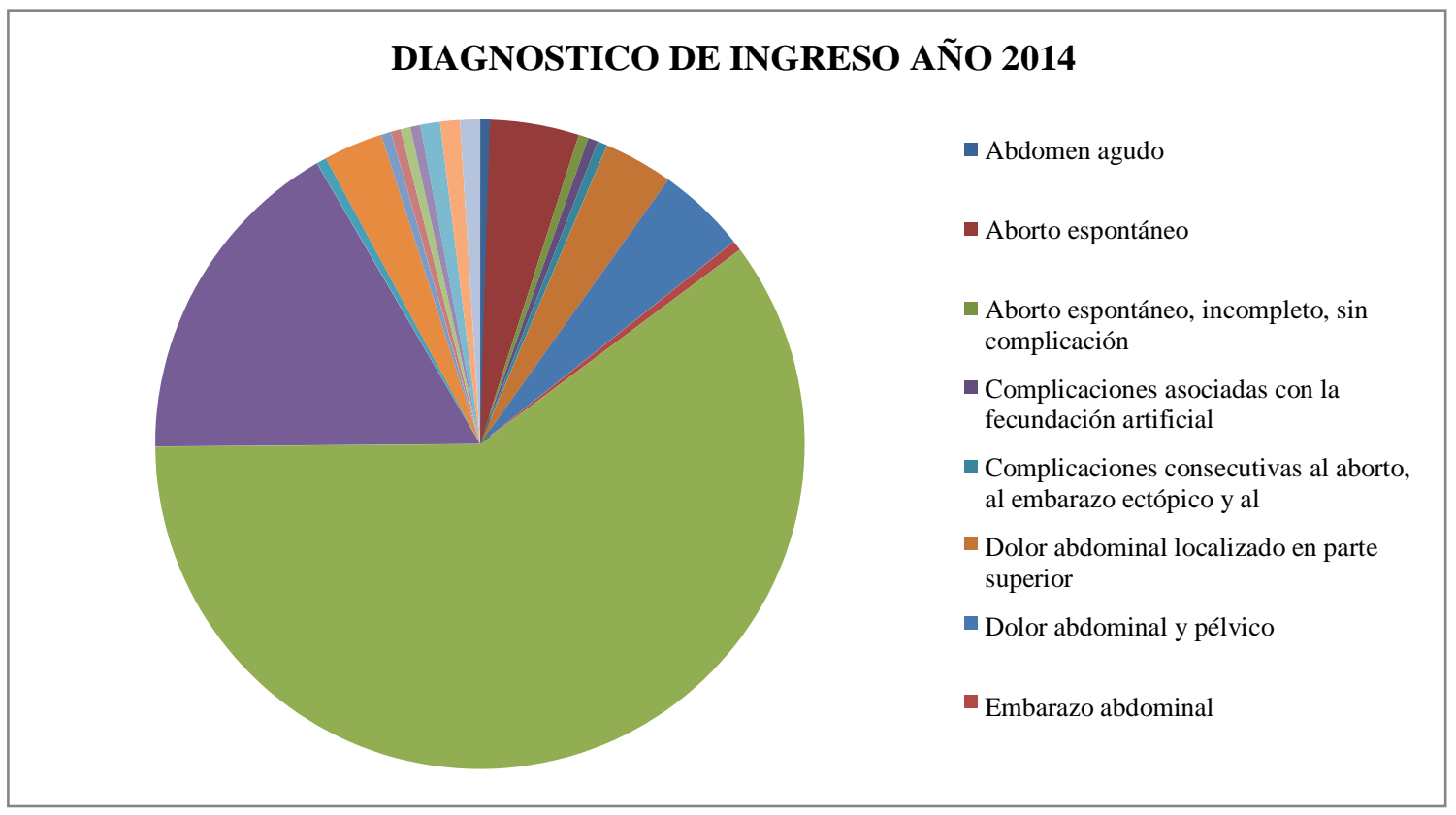

Grafico $N^{\circ}$ 5.- Diagnóstico de ingreso

- Abdomen agudo: $1(0,49 \%)$

- Aborto espontáneo: 9 (4,47\%)

- Aborto espontáneo, incompleto, sin complicación: $1(0,49 \%)$

- Complicaciones asociadas con la fecundación artificial:1 (0,49\%)

- Complicaciones consecutivas al aborto, y al al embarazo ectópico: 1 (0,49\%)

- Dolor abdominal localizado en parte superior: $7(3,48 \%)$

- Dolor abdominal y pélvico: $9(4,47 \%)$

- Embarazo abdominal: $1(0,49 \%)$

- Embarazo ectópico: 122 (60,69\%)

- Embarazo ectópico, no especificado: 34 (16,91\%)

- Embarazo tubárico: $1(0,49 \%)$

- Leiomioma del útero: 6 ( $2,98 \%)$

- Mola hidatiforme : $1(0,49 \%)$

- Otras hemorragias uterinas o vaginales anormales: $1(0,49 \%)$

- Otro trauma obstétrico: $1(0,49 \%)$

- Otros partos únicos por cesárea: $1(0,49 \%)$ 
- Parto por cesárea de emergencia : 2 (0,99\%)

- Parto único por cesárea: $2(0,99 \%)$

- Preeclampsia severa: $2 \quad(0,99 \%)$

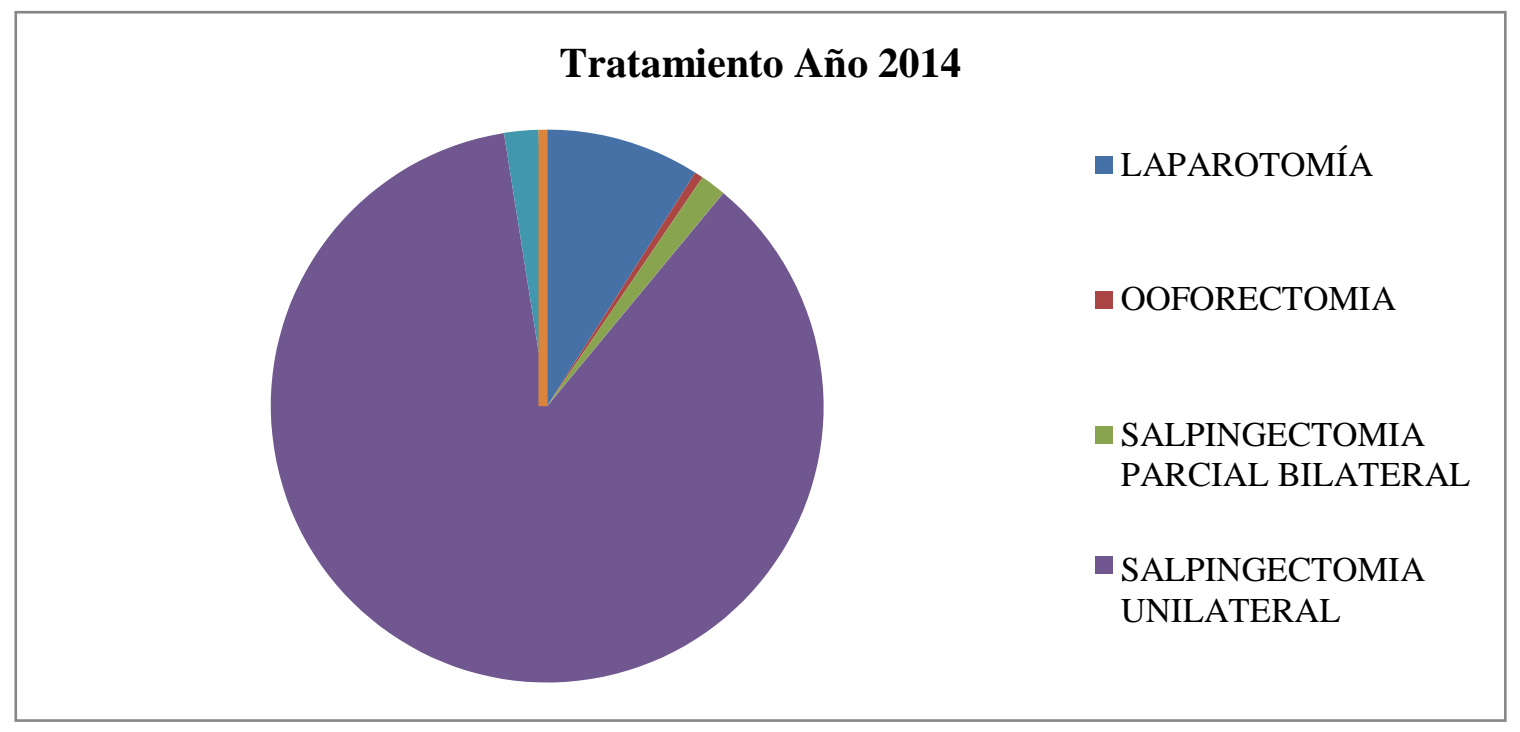

Grafico $N^{\circ}$ 6.- Tratamiento Quirúrgico

- Laparotomía: 18 (9\%)

- Ooforectomia: 1 (1\%)

- Salpingectomia parcial bilateral : $3(2 \%)$

- Salpingectomia unilateral: 173 (87\%)

- Salpingectomia unilateral laparoscopica: 4 (1\%)

- Salpingostomia: $1(0 \%)$ 


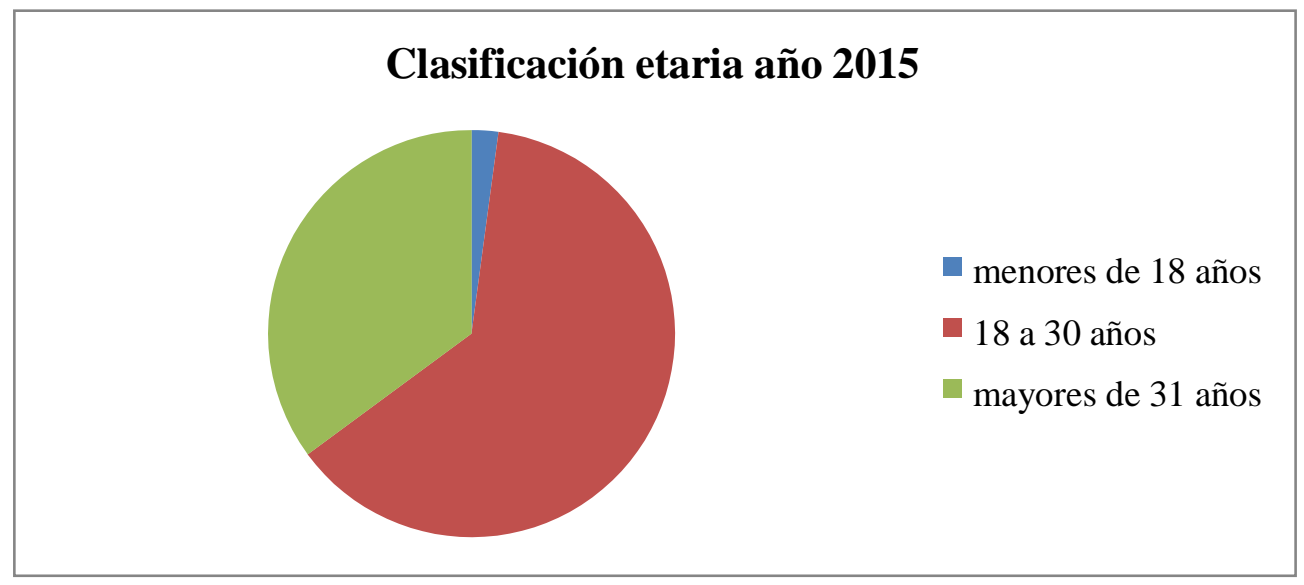

Grafico $N^{\circ}$ 7.- Grupo etario

- 4 son menores de 18 años corresponde al 2\%,

- 118 estan entre los 18 a 30 años de edad corresponde al 63\%,

- 66 son mayores de 31 años, corresponde al 35\%

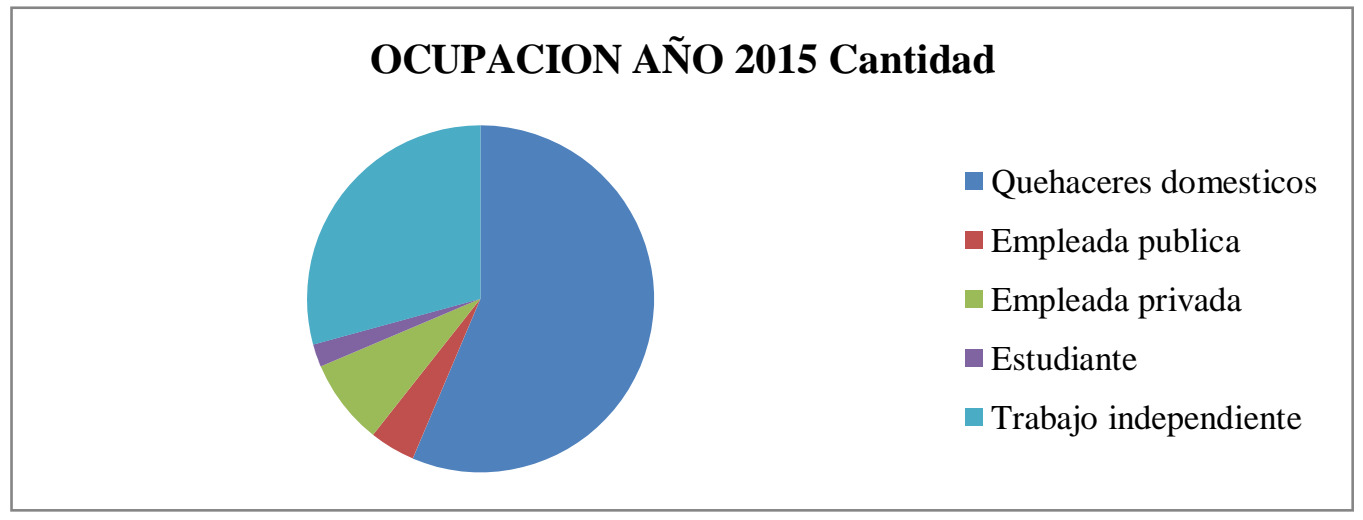

\section{Grafico $N^{\circ}$ 8.- Ocupación}

- Quehaceres domésticos: 106 (56\%)

- Empleada pública: 8 (4\%)

- Empleada privada: $15(8 \%)$

- Estudiante: 4 (2\%)

- Trabajo independiente: 55 (29\%) 


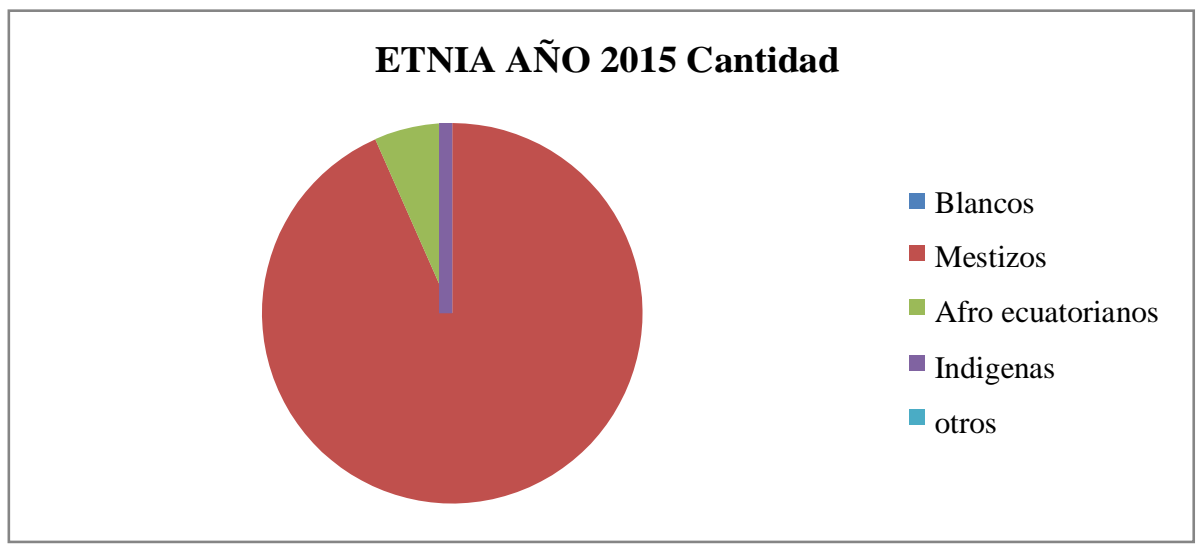

Grafico $N^{\circ}$ 9.- Etnia

- Blancos: $0(0 \%)$

- Mestizos $176(94 \%)$

- Afro ecuatorianos $10(5 \%)$

- Indígenas $2(1 \%)$

- Otros: $0(0 \%)$

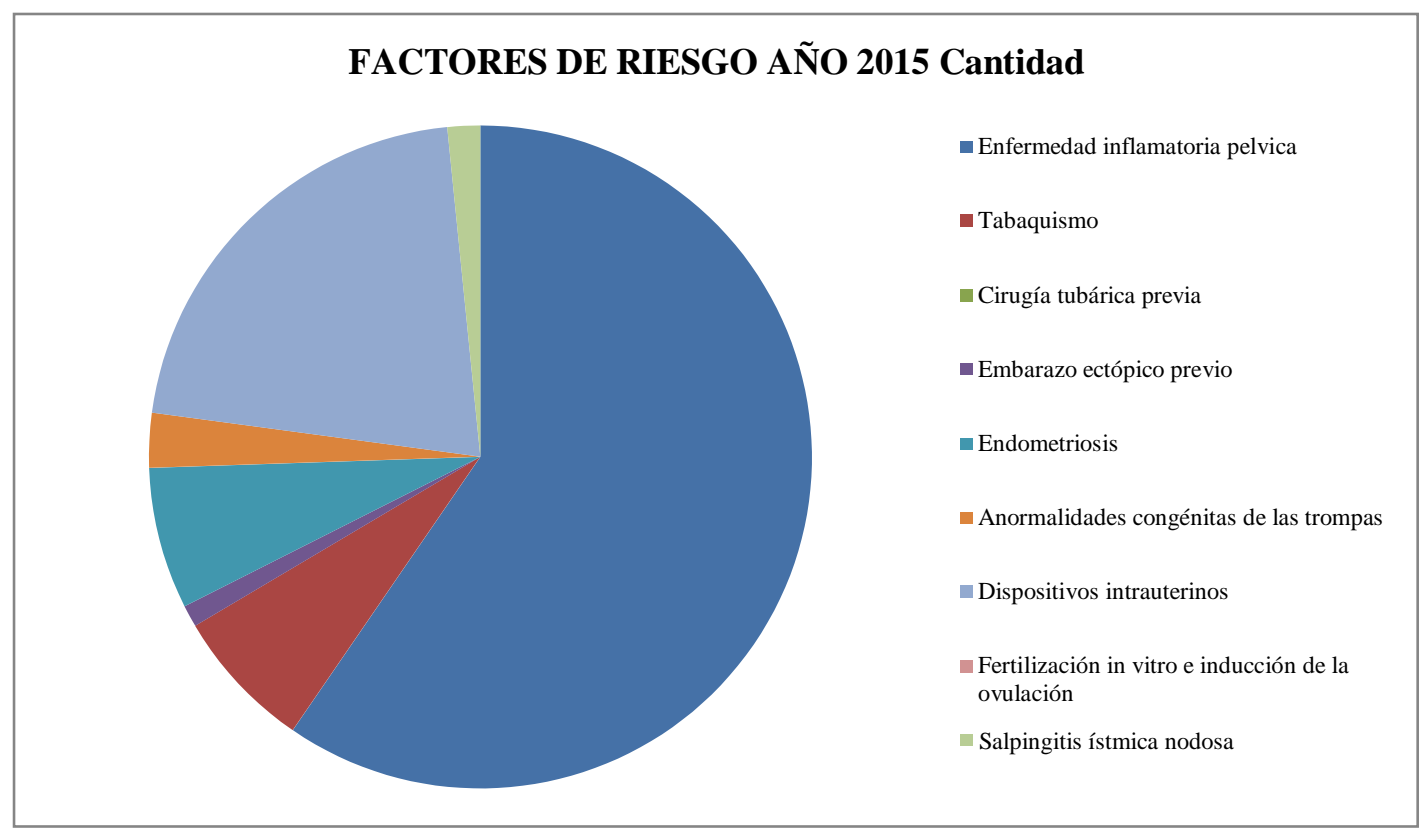

Grafico $N^{\circ}$ 10.- Factores de riesgo 
- Enfermedad inflamatoria pelvica: $112(60 \%)$

- Tabaquismo: $13(7 \%)$

- Cirugía tubárica previa: $0(0 \%)$

- Embarazo ectópico previo: 2 (1\%)

- Endometriosis: 13 (7\%)

- Anormalidades congénitas de las trompas: 5 (3\%)

- Dispositivos intrauterinos: $40(21 \%)$

- Fertilización in vitro e inducción de la ovulación: $0(0 \%)$

- Salpingitis ístmica nodosa: $3(2 \%)$

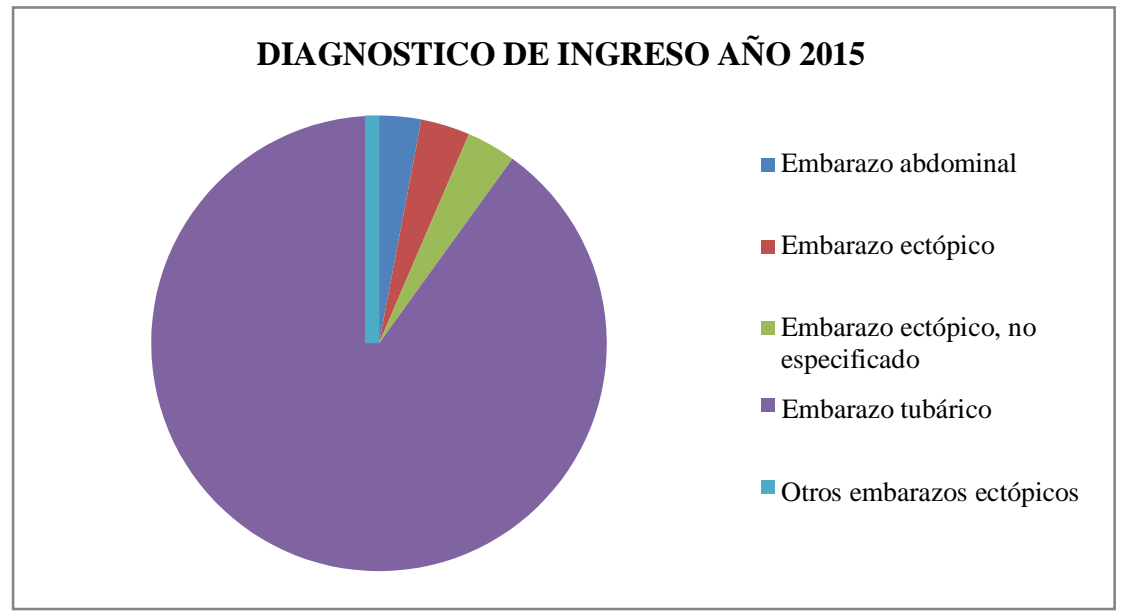

Grafico $N^{\circ}$ 11.- Diagnóstico de ingreso

- Embarazo abdominal: 6(3\%)

- Embarazo ectópico: 7 (3\%)

- Embarazo ectópico, no especificado: 7 (3\%)

- Embarazo tubárico: 179 (89\%)

- Otros embarazos ectópicos: $2(1 \%)$ 


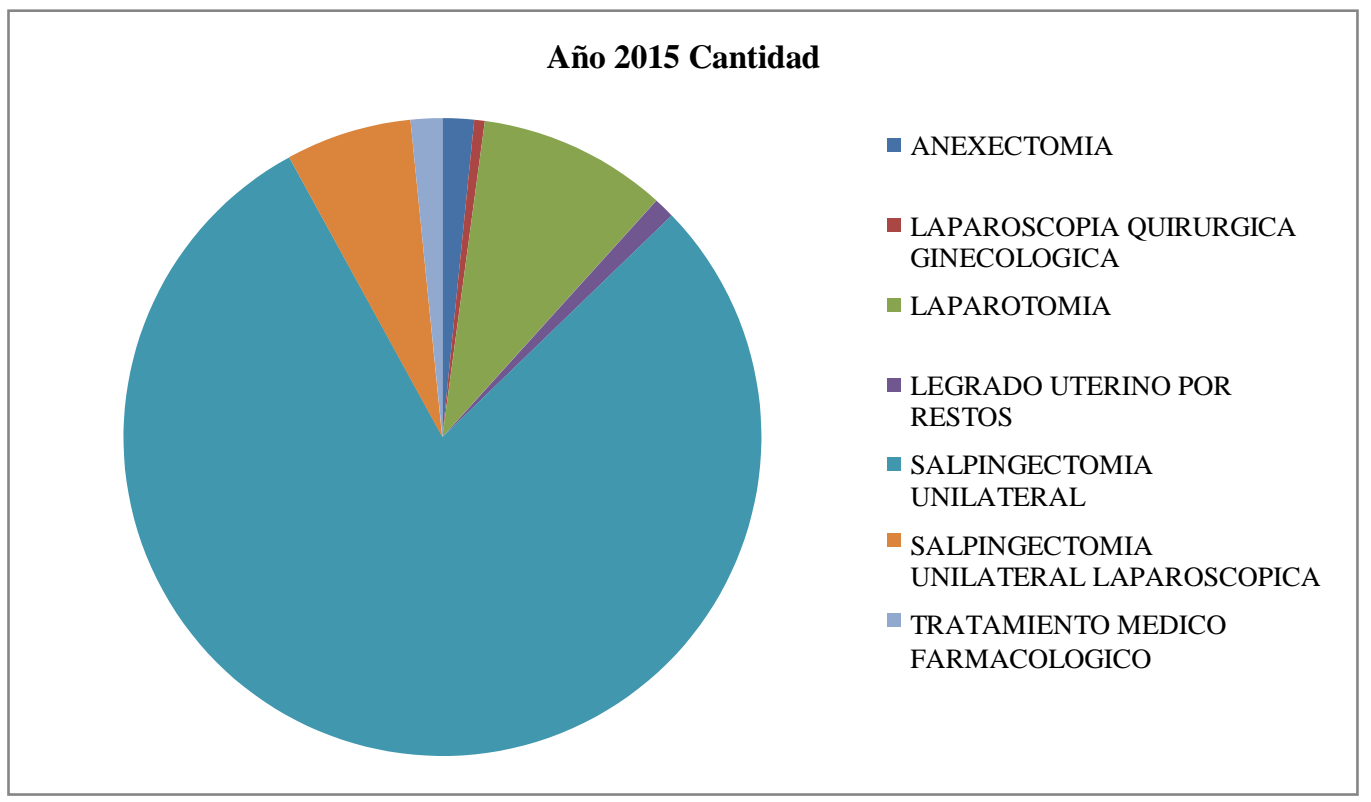

\section{Grafico $N^{\circ}$ 12.- Tratamiento médico farmacológico}

- Tratamiento médico farmacológico: 3 ( 2\%)

- Anexectomía: $3(2 \%)$

- Laparoscopía quirúrgica ginecológica: 1 (1\%)

- Laparotomía: 18 (10\%)

- Legrado uterino por restos: $2(1 \%)$

- Salpingectomía unilateral: 149 ( $79 \%$ )

- Salpingectomía unilateral laparoscópica: $12 \quad$ (6\%)

\section{Conclusion.}

En base a la investigación de las historias clínicas y la correlación con la revisión bibliográfica sobre el tema debo señalar lo siguiente:

- En el estudio etario el grupo de mayor riesgo es el de 18 a 30 años, la etnia que más resulto afectada fue la mestiza en el año 2014 con el 94\% y en el año 2015 93\%, se correlaciona con el estudio realizado en Cuenca en el año 2014 ${ }^{7}$. 
- el nivel educacional en su gran mayoría resulto ser básico a secundario y la ocupación de la mayoría es ama de casa.

- La causas más frecuente que están en relación con el embarazo ectópico es la enfermedad inflamatoria pélvica y/o las infecciones a este nivel. En nuestro país los medios de reproducción asistida no consisten en si en un alto índice de casos debido a que no es aún muy popular y/o porque los costos son prohibitivos para muchas parejas que no pueden tener hijos y desean optar por este recurso. Así mismo la relación con el índice tabáquico es menor ya que afortunadamente esta tendencia al tabaquismo es aun pequeña, sin embargo en un futuro próximo puede ser un factor de riesgo muy grande.

\section{Recomendaciones.}

En base a la investigación realizada se sugieren las siguientes recomendaciones:

1. Dentro de las campañas de educación sexual se debe incluir y concientisar a todo hombre y mujer en edad reproductiva sobre el embarazo ectopico, el mismo que es el que ocupa el numero 1 de mortalidad materna en el primer trimestre de embarazo.

2. Se debe diagnosticar y dar a conocer los factores de riesgos que ya hemos mencionada previamente.

3. Es importantre si tiene vida sexual activa y sus ciclos normales, si cursa con amenorrea asistir a la consulta y descartar un embarazo intra o extra uterino. Y si es posible investigar ecosonograficamente si ai saco gestacional y embrion dentro del utero. O si no lo hay visualizar en otro lugar, y hacer su relacion con el estudio cuantitativo de la b-hcg 
4. Usar el algorritmo diagnostico que permite al medico de emergencia o consulta externa identificar un embarazo ectopico y lo pueda referir al especialista para realizar su obtimo manejo.

5. El manejo correcto del tratamiento para embarazo ectopico, constituye una disminucion de riesgo materno y de perdida de la fertilidad de esta paciente.

\section{Bibliografia.}

1. Cepeda-Silva A. Abdomen agudo en el embarazo: caso clínico comentado. Perinatología y reproducción humana. 2013 Mar; 27(2): p. 123-128.

2. Kilpatrick C, Monga M. Abordaje del abdomen agudo en el embarazo. Obstet Gynecol Clin N Am. 2007; 34(1): p. 389-402.

3. Metter L, Schollmeyer T, Akatout I. Adhesions during and after surgical procedures, their prevention and impact on women health. Whe. 2012; 8(1): p. 495-498.

4. F. Gary Cunningham, Kennneth J. leveno. Wiliams obstetricia. 23rd ed. Mexico: Mc Graw Hill; 2011.

5. Usiña J, Céspedes D, Yunga J. Anuario de Estadísticas Vitales - Nacimientos y Defunciones. Quito: INEC, DIRECCIÓN DE ESTADÍSTICAS SOCIDEMOGRÁFICAS; 2014.

6. Oscar Andrés F, Olaya N. Embarazo ectópico esplénico: reporte de caso y revisión de la literatura. Revista Colombiana de Obstetricia y Ginecología. 2009 Ene; 60(1): p. 89-91.

7. Morales-Zambrano C, Nagua-Blanca D. Caracteristicas clínicas y factores de riesgodel embarazo ectopicoen el hospital Vicente Corralmoscoso de la ciudad de cuenca durante el periodo 208 2012. Tesis previo a la obtencion de Medico General. Universidad de Cuenca, Escuela de Medicina; 2014. 